Pov. Chil. Pediats. $63(4) ; 177 \cdot 183,1992$

\title{
Ductus arterioso persistente y estado carencial de cobre
}

\author{
Francisco Alliende G. ${ }^{1}$; Ignacio Villa-Elizaga ${ }^{1}$; \\ Federico Antillón $\mathrm{K}^{\mathrm{t}}$ \\ Patent ductus arteriosus and copper-deficiency
}

\begin{abstract}
To investigate the efects of copper-deficiency on spontaneous closure of ductus arteriosus in newborn rats. an experimental model consisting in three groups of ten female Sprague-Dowley rats each was used. Group 1 rats, copper-deficient, were fed a copper-free diet from 35 days before pregnancy until birth. Group 11. control rats, were given a diet containing appropriate amounts of copper (6 ppm) and energy. Group III, the pair-fed rats, received a diet that was adecuate in copper content but restricted in energy to the same caloric ingestion previously shown by copper deprived rats. The ductus arteriosus of 10 newborn rats of each group were studied by hystological methods at sometime between the third and twelfth hour of life, and it was patent in 100\%, 20\% and $40 \%$ of the cases of copper deprived, control and pair fed rats respectively (Chi square test, $p=0.00091$. The wall thickness of patent ductus from copper deprived rats were significantiy lower than those from rats of the other two groups (K.ruskall-Wallis Test; $\rho=0.0079$ ). Our results suggest that materno-fetal copper deficiency significantly af fects the spontaneous closure rate of the ductus arteriosus in the rat. The mechanism of this abnormal response probably includes structural changes in the ductal wall, alterations in the energy vield process or abnormal reguletion of prostaglandins E2 and F2 - $\alpha$ synthesis.
\end{abstract}

(Key words: Patent ductus arteriosus, copper-deficiency, rats.)

El significado biológico del cobre fue evidenciado por primera vez en el año 1928 cuando Hart y cols. demostraran su esencialidad en la sintesis de hemoglobina en la rata ${ }^{1}$. No obstante, no fue sino hasta el año 1956 cuando se describió por primera vez un estado carencial en el ser humano ${ }^{2}$. Desde entonces, numerosas publicaciones tratan sobre estados de deficiencia de cobre, con especial referencia a la prematuridad, síndromes de malabsorción y nutrición parenteral $^{3-9}$. El progreso en los conocimientos relativos al metabolismo del cobre y sus requerimientos en diferentes situaciones no ha impedido que continúen apareciendo publicaciones con casos clínicos de la deficiencia, como muestra de que el problema aún está vigente ${ }^{10-13}$.

La enfermedad cardíaca producida por una situación experimental de déficit de cobre ha sido caracterizada por hipertrofia y palidez del

1. Unidad de Investigación Pediátrica, Departamento de Pediatría, Facultad de Medicina, Universidad de Navarra, Espara. miocardio, también por zonas de necrosis, fibrosis, aneurismas, e incluso perforaciones ${ }^{14}$. Trabajos posteriores evidenciaron desestructuración mitocondrial, déficit enzimáticos, disminución de la concentración de ATP y fosfocreatina ${ }^{15}$. Estas alteraciones, que en forma global se aseme. jan a aquellas encontradas en hipoxia crónica, no se asociaron a lesiones vasculares concomitantes, por lo que se consideró obedecían a un defecto primario del miocardio y fueron interpretadas como constituyentes de una miocardiopatía cobredependiente ${ }^{14,15}$.

La importancia del cobre en la normal formación de la pared de los vasos sanguíneos fue puesta de manifiesto en los años 60 , al comprobarse su relación con la síntesis de elastina, en función de la actividad de la enzima lisil-oxidasa ${ }^{16-20}$.

Un aspecto más reciente de las consecuencias del déficit de cobre en el ser humano fue puesto en evidencia por Sutton en 1985, al describir cuatro prematuros con ductus arterioso permeable, reversible únicamente a la administración de cobre ${ }^{21}$. Este hallazgo no ha sido objeto de mayor estudio, desconociéndose actualmente 
los mecanismos que en ello pudieron haber participado.

En base a estas últimas observaciones nos hemos propuesto desarrollar un modelo experimental que determine la real relación entre déficit nutricional de cobre y persistencia del ductus arterioso. Con los resultados obtenidos, junto a los conocimientos actuales, nos permitiremos esbozar una hipótesis fisiopatológica que expli. que tal asociación.

\section{Material y Método}

Se utilizaron 30 ratas hembras vírgenes, cepa Sprague-Dowley con peso entre 110 y $130 \mathrm{~g}$ (Panlab. Barcelona) que fueron colocadas en jaulas individuales de acero inoxidable en un cuarto con temperatura, haz, ventilación y humedad controladas $\left(21+2^{\circ} \mathrm{C}\right.$, ciclo de luz-oscuridad de $12 \mathrm{~h}, 50 \%$ de humedad). Utilizamos dietas purificadas en polvo, "control" o normocúprica (cobre $=6 \mathrm{ppm})$ y cuprodeficiente $(00 \mathrm{bre}=0 \mathrm{ppm})$, adquiridas en la casa Dyets Inc. Bethehem, Pensilvania, EE.UU. (referencia 100000 y 115501 , respectivamente, del catálogo de dietas de la misma casa, edición 1989-1990). Todas ellas fueron elaboradas de acuerdo a la normativa del "American Institute of Nutrition" $(\text { AIN })^{22,23}$. Las ratas fueron aclimatadas durante un período de 5 dias, en el cual todas fueron alimentadas con la dieta "normocúprica". Segujdamente fueron distribuidas aleatoriamente en tres grupos (n: 10 ) que recibieron, respectivamente, una đieta carente de cobre, ad libium (grupo cuprodeficiente): dieta normocúprica ad libitum (grupo control) y dieta normocúprica con. ingesta reducida de acuerdo a la cantidad recibida por el grupo cuprodeficiente (grupo "pair fed"). A los tres grupos se les suministró agua desionizada (Milli-RO4, Millipore Corp.). Antes del apareamiento las ratas se mantuvieron con la dieta experimental durante 35 $d$ ías (período pregestacional), Una yez finalizada da gestación de tres semanas, tras el parto, se inició el período neonatal del estudio. En esta última etapa, entre la $3^{\mathrm{a}}$ y $12^{\mathrm{a}}$ h de vida, fueton aralizados los tejidos maternos y neonatales. Las ratas fueron pesadas al iniciar el período de aclimatación y la dieta experimental, luego cada 10 días durante la fase pregestacional y diariamente durante la gestación.

Las muestras de sangre fueron obtenidas del plexo retronrbitario con la ayuda de un capilae de microhe matocrito heparinizado, bajo anestesia superficial con $e^{\text {éter }}{ }^{34}$. La determinación de hematocrito se llevó a cabo cada 10 días en la etapa pregestacional y los dias cero, 11 y 21 de la etapa gestacional. El cobre plasnático fue deteminado cada 10 días en la etapa pregestacional y las días $0,11,21$ de la etapa gestacional. Las muestras fueron analizadas en un espectofotómetro de absorción atónica de llama (GBC 2000, Australia), previa dilución 1:3 en agua desionizada (Merck). El colesterol plasmático fue medido los días 10 y 30 de la etapa pregestacional y días 0 y 21 de la etapa gestacional, mediante método enzimático acoplado a la oxidación de p-amino fenazona (Boehringer Mantheim), auto- matizado, en un equipo discreto selectivo (Hitachi 704). Se obtuvjeron lambién muestras tisulares de las ratas madres (hígado, riñón, bazo, cerebro, corazón, pulmón y músculo) y de su descendencia (hígado, riñón, cercbro y corazón), para el análisis de cobre. El material procedente de la descendencia fue obtenido de 5 recién nacidos por cada camada (n; 50). Los óngânos abtenidos fueron lavados con solución de $\mathrm{NaCl}$ al $90 \% 0$, pesados y almacenados en tubos de poliesti reno en un congelador $\mathbf{a}-70^{\circ} \mathrm{C}$ hasta el día de su análisis. La determinación de $\mathrm{Cu}$ fue llevada a cabo empleando $0,1 \mathrm{~g}$ de muestra previamente secada entre 40 a $60^{\circ} \mathrm{C}$ durante 6 a $8 \mathrm{~h}$, digerida con $0,5 \mathrm{ml}$ de ácido nítrico $16 \mathrm{~N}$ (Suprapur, Merck) y diluida en $1 \mathrm{ml}$ de agua desionizada. Las soluciones obtenidas fueron leidas en un espectrofotometro de absorción atómica de llama, como el que ya se ha mencionado.

Microscopia óptica. El estudio del ductus arterioso se realizó en diez recién nacidos de cada grupo. Para este efecto se eligió al azar una rata recién nacida de 3 a 12 h de vida de cada camada. El procedímiento se realizó en preparaciones histológicas de cortes frontales de $10 \mu$ de los bloques torácicos teñidos con hematoxilina-eosina. Una vez analizados todos los cortes se seleccionaron aquellos planos que coincidían con el ductus arterioso, tras la emergencia de las arterias pulmonares. On un microscopio (Olympus), dotado de ocular reglado (Leitz) y previa calibración con micrómetro de $100 \times 0,1 \mathrm{~mm}$ (Graticules Ltd.), fue medido el diámetro menor de la luz y el grosor de la pared del ductus arterioso. Se consideró que un ductus persistía permeable cuando el diámetro de la luz superaba los $100 \mu \mathrm{m}$

Análisis estadístico. Para comparar los resultados entre los tres grupos se utilizó el análisis de la varianza (ANOVA) de un criterio de clasificación y la prueba a posteriori de Scheffe $(\alpha=0,05)$. Cuando no se cumplieron las condiciones necesarias para realizar esta prueba paramétrica, se utilizó el equivalente no paramétrico Kruskal-Wallis $(\alpha=0,05)$. Para estudiar las variables cualitativas (ductus cerrado/ductus permeable), se realizó un análisis de contingencia aplicando la prueba de Chi cuadrado.

\section{Resultados}

El crecimiento de las ratas fue influido por la dieta. En los grupos deficientes de cobre y de alimentación pareada el peso era significativamente menor que en el grupo control $(\mathrm{p}<0,01)$ desde el día 28 de la etapa pregestacional. No hubo diferencias significativas entre los pesos del grupo privado de cobre y el de alimentación pareada (figura 1). La ingesta de la dieta suministrada fue significativamente menor en el grupo privado de cobre que en las ratas controles, desde los primeros días de la experimentación. La diferencia fue significativa desde la segunda semana de la fase pregestacional $(p<0,005)$ hasta la primera de la fase gestacional. Como era de 


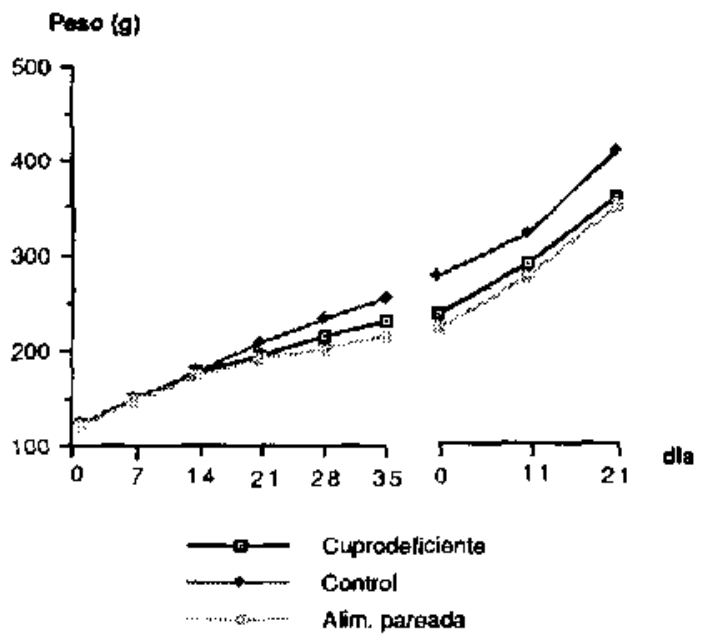

Figura 1: Evolución semanal del peso, durante los períodos pregestacional y gestacional de las ratas deprivadas de cobre, con alimentación normal y alimentación pareada.

esperar, la ingesta alimentaria de los grupos cuprodeficiente $y$ de alimentación pareada no mostró diferencias significativas (figura 2).

En los animales con dieta sin cobre se registró una rápida caída de las concentraciones plasmáticas del metal, siendo significativa la diferencia observada con respecto a los grupos control y de alimentación pareada desde el $10^{\circ}$ dia de la fase pregestacional ( $p<0,001$ ). No se obseryaron diferencias significativas entre los gnipos control y alimentación pareada. La concentración de cobre en los órganos maternos analizados fue significativamente inferior en el grupo cuprodeficiente que en los grupos control y de alimentación pareada ( $\mathbf{p}<$ 0,001 ), sin que se hayan encontrado diferencias entre estos últimos. La concentración de colesterol plasmático durante la etapa pregestacional se elevó significativamente en el grupo con dieta deficiente en cobre, lo que determinó que alcanzara valores significativamente más elevados que el grupo de alimentación pareada. Durante la fase gestacional no se apreciaron variaciones significativas ni diferencias entre los tres grupos. En las ratas sometidas a dieta sin cobre se registró una caída significativa del hematocrito, desde el día 30 de la fase pregestacional ( $\mathrm{p}<0,001$ ), manteniéndose una diferencia de igual grado durante la gestación, etapa durante la cual los tres grupos mostraron una moderada disminución de los valores.

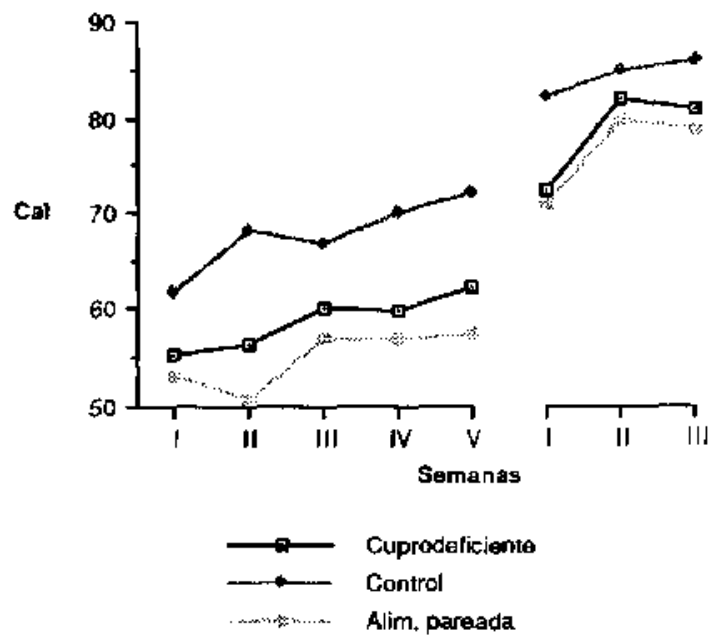

Figura 2: Ingesta diaria promedio a lo largo de las distintas semanas de los períodos pregestacional y gestacional.

En la exploración general de los recién nacidos, todos los de] grupo cuprodeficiente presentaban hematomas subcutáneos, laxitud y sequedad de la piel. Algunos mostraban también dificultad respiratoria moderada y movjmientos anormales. Las concentraciones de cobre en los tejidos analizados eran significativamente más bajas en los recién nacidos del grupo deficiente en cobre que en los grupos controly de alimentación pareada (tabla 1). La comparación entre estos últimos dos no reflejó diferen. cias significativas. No se observaron malformaciones en el corazón o grandes vasos en la inspección con estereomicroscopio. El estudio del ductus arterioso bajo microscopio de luz (figura 3) evidenció que éste permaneció abierto en $100 \%$ de los recién nacidos analizados $(10 / 10)$ del grupo con dieta deficiente en cobre, en $20 \%$ (2/10) del grupo control y en el $40 \%(4 / 10)$ del grupo de alimentación pareada, valores significativamente diferentes de acuerdo al resultado de la prueba de Chi-cuadrado $(\mathrm{p}<0,0009)$. Al comparar la persistencia del ductus en recién nacidos de madres deprivadas de cobre con los de alimentación pareada se comprueba $\mathrm{p}=$ 0,0034 ; entre los primeros $y$ controles $p$ $=0,0003$, mientras que entre nacidos de animales controles y con alimentación pareada el resultado es $\mathrm{p}=0,329$. En la tabla 2 se expresan los promedios, desviaciones estándar y el análisis estadístico de las mediciones del grosor de 
Tabla 1

Concentraciones de cobre $(\mu \mathrm{g} / \mathrm{g})$ en los tejidos de fatas recjén nacidas de madres con dieta ad libitum deficiente en cobre (D), dieta normal pareada en calorías a la ingerida por las ratas deprivadas de cobre (P) y normal ad libitum (C)

\begin{tabular}{|c|c|c|c|c|c|c|c|}
\hline & $\underset{(\mu \mathrm{g} / \mathrm{g})}{\mathrm{D}}$ & $\underset{\left(\mu_{\mathrm{g}} / \mathrm{g}\right)}{\mathrm{C}}$ & $\underset{(\mu \mathrm{g} / \mathrm{g})}{\mathbf{P}}$ & $\begin{array}{l}\text { Anova } \\
\text { (p) }\end{array}$ & $\mathrm{D} / \mathrm{C}$ & $\begin{array}{r}\text { Scheffe } \\
\mathrm{D} / \mathbf{P}\end{array}$ & $\mathrm{C} / \mathrm{P}$ \\
\hline Higado Cobre & $0,490 \pm 0,130$ & $16,210 \pm 3,430$ & $14,460 \pm 4,180$ & 0,0001 & * & * & ns \\
\hline Riñón Cobre & $0,520 \pm 0,150$ & $1,330 \pm 0,180$ & $1,280 \pm 0,190$ & 0,0001 & $*$ & * & ns \\
\hline Cerebro Cobre & $0,117 \pm 0,034$ & $1,500 \pm 0,178$ & $1,431 \pm 0,186$ & 0,0001 & $*$ & $*$ & $\mathrm{~ns}$ \\
\hline Corazón Cobre & $0,583 \pm 0,167$ & $2,461 \pm 0,400$ & $2,222 \pm 0,356$ & 0,0001 & * & * & ns \\
\hline
\end{tabular}

* significativo al $99 \%$.
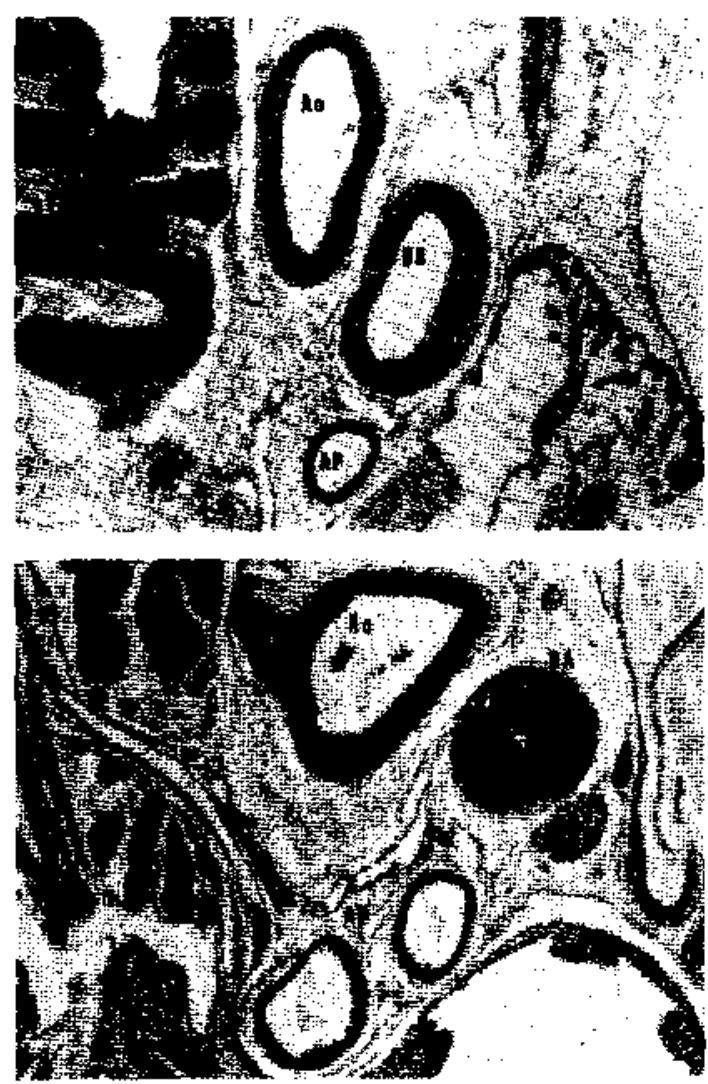

Figura 3: Microfotografía de un corte histológico frontal de tórax neonatal que pasa por un plano coincidente con el ductus arterioso (DA), emergencia de las arterias pulmonares (AP) y cayado aórtico (Ao). La foto superior corresponde a un recién nacido del grupo cuprodeficiente en donde se distingue la amplia luz de DA. En la foto inferior, contral, se observa un aspecto del ductus contraído $(40 \mathrm{x})$. la pared y la luz, sólo de los ductus encontrados permeables en los tres grupos, mostrando que el grosor de la pared ductal era significativamente inferior en los ductus permeables del grupo cuprodeficiente, que en aquellos tambien permeables de los grupos control y alimentación pareada, siendo similar en todos ellos el diámetro de la luz.

\section{Discusión}

En situación fisiológica, el ductus de las ratas se cierra espontáneamente dentro de los primeros 180 minutos de vida postnatal ${ }^{25}$, lo que se asocia con cambios estructurales que comienzan a los 60 minutos ${ }^{26}$. En nuestro caso los neonatos fueron estudiados entre las tres $y$ doce horas de vida, lo que ha permitido, por una parte, consignar las manifestaciones clinicas de interés tras algunas horas de observación y, por otra, observar si ocurre el cierre del conducto o, al menos, aparición de evidencias de que pudiera ocurrir. En estas circunstancias bastaría la confirmación de la permeabilidad del ductus para considerarla un hecho patológico. El análisis morfométrico, basado en los procedimientos utilizados por Hörnblad ${ }^{27}$, de aquellos ductus que persistieron permeables tanto del grupo deprivado de cobre como en los otros dos, permitió reforzar las primeras impresiones, con la identificación de diferencias morfológicas significativas en el grosor de pared de conductos igualmente permeables. A pesar de que la pared del ductus es pobre en fibras de colágeno 
Tabla 2

Grosor de la pared y luz (um, $\bar{x} \pm$ DE) del ductus en ratas recién nacidas provenientes de madres con dietas ad libirum deficiente de cobre (P), nomal pareada en calor jas a la ingerida por ratas deprivadas de cobre (P) y normal od libitum (C)

\begin{tabular}{lcccc}
\hline & $\begin{array}{c}\mathrm{D}(\mathrm{n}: 10) \\
\overline{\mathrm{x}} \pm \mathrm{DE}\end{array}$ & $\begin{array}{c}\mathrm{C}(\mathrm{n}: 2) \\
\overline{\mathrm{x}} \pm \mathrm{DE}\end{array}$ & $\begin{array}{c}P(n: 4) \\
\tilde{\mathrm{x}} \pm \mathrm{DE}\end{array}$ & $\mathrm{p}^{*}$ \\
\hline Pared & $-69,1 \pm 17,8$ & $117,6 \pm 1,4$ & $96,3 \pm 5,7$ & 0,0079 \\
Luz & $274,5 \pm 84,0$ & $215,3 \pm 97,7$ & $252,8 \pm 26,2$ & 0,5304 \\
\hline
\end{tabular}

* p según prueba de Kruskall Wallis.

y elastina ${ }^{28}$, es probable que la disminución de su grosor esté en relación con desestructuración y disminución de ellas, fruto de la menor actividad de la enzima cuprodependiente lisiloxidasa. Esto, sin embargo, no explica totalmente que el ductus persistiera abierto en al grupo deficiente en cobre, dado que el cierre, en condiciones fissológicas, parece depender principalmente de la contracción de la musculatura lisa de la túnica media ${ }^{25,28}$.

Se sabe que las variaciones de las concentraciones sanguineas de prostaglandinas ( $\mathrm{PG}$ ) y oxígeno tienen un importante papel en el cierre fisiológico del ductus ${ }^{29-32}$. Mientras las prostaglandinas de clase $\mathbf{E}$ tienen propiedades relajadoras del ductus, la $\mathrm{F}_{2}-\alpha$ es la única con propiedades constrictoras ${ }^{33}$, si bien es cierto que a esta última se la ha desestimado en el mecanismo de cierre fisiológico. Trabajos en preparaciones tisulares han evidenciado importante participación del cobre en la regulación de la sintesis de prostaglandinas, con potente efecto inhibidor sobre la de $E_{1}$, a la vez que estimulador en la de $\mathrm{F}_{2}-\alpha$. Se ha sugerido que el metal actuaria a través de la inactivación de la enzima endoperóxido- $\mathrm{E}_{2}$ isomerasa, con activación concomitante de la endoperóxido- $\mathrm{F}_{2}-\alpha-$ reductasa $^{34}$. Este hecho tiene enorme importancia si consideramos que a las $P G E_{2}$ y $P G F_{2}-\alpha$ se les conocen efectos contrapuestos sobre la musculatura del ductus. Es probable que en nuestros neonatos la deficiencia de cobre haya anulado la regulación propuesta por los autores antes mencionados, evitando en parte que se produzca la disminución fisiológica de la $\mathrm{PG} \mathrm{E}_{2}$ o el aumento de la $P G F_{2-\alpha}$ o ambos.

El déficit de cobre provoca en el corazón una miocardiopatía, que podría guardar relación con alguna alteración en el metabolismo energético y, además de modificaciones estructurales, puede causar trastomos electrofisiológicos ${ }^{35}$ y mecánicos ${ }^{36}$, no siendo posible descartar la probabilidad de que ellos también puedan ocurrir en la musculatura ductal, con efectos negativos sobre su contractilidad. Esto adquiere especial relevancia si consideramos la hipótesis propuesta por $\mathrm{Fay}^{37}$, que atribuye el papel constrictor del oxígeno a una estimulación de la síntessis de ATP. Finalmente, la deficiencia de cobre afecta la integridad estructural del pulmó $^{38}$, alterando de esta forma la función de intercambio gaseoso, evitándose una adecuada oxigenación de los tejidos.

Concluimos que la cuprodeficiencia efectivamente tiene un papel importante en el cierre del ductus arterioso en el recién nacido. El déficit de cobre, sobre la base de una alteración estructural de la pared del vaso, produciría una alteración en la regulación de la síntesis de prostaglandinas, un bajo aporte de oxígeno y una disminución de la capacidad de respuesta contráctil, que en conjunto determinarian que el đuctus arterioso continúe permeable.

\section{Resumen}

Con el propósito de estudiar el efecto de la privación del cobre en la dieta sobre el cierre del ductus arterioso se estudiaron tres grupos de ratas hembras Sprague-Dowley de 10 animales cada uno. Al grupo I se le privó de dicho nutriente, al grupo II se le dio una dieta normal y al grupo IiI se le restringieron las calorías a la ingesta mostrada por las del primer grupo, pero con aporte normal de cobre, desde 35 dias antes de la gestación y a lo largo de ésta, logrando un estado de deficiencia de cobre en las madres gestantes y la descendencia del primer grupo. Se 
hicieron estudios morfométricos en 10 recién nacidos de cada grupo, uno de cada camada. En todos los recién nacidos del grupo cuprodeficiente $(10 / 10)$, en $20 \%(2 / 10)$ del grupo control, $y$ en $40 \%(4 / 10)$ del grupo de alimentación pareada, el ductus arterioso permaneció permeable, diferencias estadisticamente muy significativas de acuerdo a la prueba de Chi cuadrado ( $p<0,0009$ ). El grosor de la pared, considerando sólo los ductus que permanecieron permeables en los tres grupos, fue significativamente menor en los casos deprivados de cobre que los de los otros dos grupos (Kruskall-Wallis, $p=$ 0,0079 ), mientras el diámetro de la luz era similar. La deficiencia de cobre matemofetal parece afectar significativamente el cierre espontáneo del ductus arterioso en la rata. El mecanismo por el cual esto ocurre podria incluir modificaciones estructurales de la pared del ductus, alteraciones en los procesos de obtención de energía o alteración en la regulación de la síntesis de prostaglandinas $E_{2}$ y $F_{2}-\alpha$.

(Palabras claves: Cuprodeficiencia, ductus arterioso, ratas.)

\section{Referencias}

1. Hort EB, Steenbock $H$, Wadell $J$ et al.: tron in nutrition VII. Copper as a suplement to iron for hemoglobin binding in the rat. J Biol Chem 1928; 77: 797-812

2. Sturgeon $P$, Brubaker $\mathcal{C}$ : Copper deficiency in infants. Am J Dis Child 1956;92: 254-265.

3. Karpet IT, Peden VH: Copper deficiency in longterm parenteral nutrition. J Pediatr 1972; 80 : 32-36

4. Ashkenazi A. Levin ST, Dialdetti M, Fishel E, Benvenisti $D$ : The syndrome of neonatal copper deficiency. Pediatrics 1973; $52: 525-533$.

5. Al-Rashid RA, Spangler $f$ : Neonatal copper deficiency. N Engl J Med̂́ 1971; $285: 841-843$.

6. Heller RM, Kirchner $S G$. ONeill $J A$ et al.: Skeletal changes of copper deficiency in infant receiving prolonged total parenteral nutrition. I Pediat $1978 ; 92: 947-949$.

7. Sann L, David L. Galy G, Romand-Monier M: Copper deficiency and hypocalcemic rickets in a small for date infant. Acta Paediatr Scand 1978; $67: 303-307$

8. Bennoni-Smires CH, Medina $J$, Young LW: Radiological case of the month. Am J Dis Child 1980; 134: 1155-1156.

9. Yuen P, Lin HJ, Hutchinson JH: Copper deficiency in a low birth weight infant. Arch Dis Child 1979;54:553-555.

10. Radriguez $A$, Soto $G$, Torres $S$, Venegas $G$, Castillo.Durán: Cinc $y$ cobre en pelo $y$ plasma de nivios con diarrea crónica. Acta Paediatr Scand $1985 ; 2: 849-853$.
11. Chapman $S$ : Child abuse or copper deficiency? A radiological view. Br Med J 1987;294: 1370 .

12. Velia P, Dupont D, Daoud A: La carence nutrjtionnelle en cuivse. A propos d'une observation. Ann Pediats 1989; 36: 269-274.

13. Paterson CR: Osteogenesis imperfecta and other bone disorders in differential diagnosis of unexplained fractures. Br Med J 1990;83: 72-74.

14. Kelly WA, Kesterson JW, Cariton WW: Myocadial lesions in the offspring of female rats fed a copper deficient diet Exp Mol Pathol 1974; 20 : 4056 .

IS. Kopp SU. Klevay LAT. Feliksik JM: Physiological and metabolic characterization of a cardiomyopatby induced by chronic copper deficiency. Am J Physiol 1983; 245: H855-866.

16. O'Dell BL, Hardwick BC, Reynolds $G$, Savage $J E$ : Connective tissue defect in chicks resulting from copper deficiency. Proc Soc Exp Biol Med 196I; $108 ; 402-408$.

17. Cornes WH. Shields GS, Cardwright GE, Wintrobe MM: Vascular lesions in copper-defjcient swirle. Fed Proc 1961:20: 118 (abstr).

18. Hill CHH. Starcher B. Kin CH: Role of copper in formation of elastin. Fed Proc 1967;26: 129133.

19. Simpson ChS, Jones JE, Harms RH: Ultrastructure of aortic tissue in copper-deficient and control chick embryos. J Nutr 1967;91: 283-291.

20. Pinnell $S R$, Martin $G R$ : The cross-linking of collagten and elastin; eszymatic conversion of lysine in peptide linkage to alfa-aminoadipic-gammasemialdehyde (allysine) by an extract from bone Biochemistry 1968; 61: 708-716.

21. Sutton AM, Harvie A, Cockbum F, Farquharson J. Logan RW: Copper deficiency in the pretern infant of very low birthweight. Four cases and a reference range for plasma copper. Arch Dis Child $1985 ; 60 ; 644-651$.

22. American Institute of Nutrition: Report of the AIN ad hoc Committee on Standards for Nutritional Studies. J Nutr 1977; 107: 1340-1348.

23. American Institute of Nutrition: Second Report of the AlN ad hoc Committee on Standards for Nutritional Studies. J Nutr 1980; 110:1726.

24. Stone SH: Method for obtaining venous blood From the orbital sinus of the rat or mouse. Science $1954 ; 119: 100$.

25. Janatova $T$, Jarkovska D. Hruda J, Semanek M, Ostadal B: Effect of the administration of prostaglandis ( $P G E_{2}$ ) in the laboratory rat. Physiol Bohemostov $1989 ; 38: 201-206$

26. Jarkouska D, Janatova $T$, Hruda $f$, Ostadal B, Somanek $M$ : The physiological closure of ductus asteriosus in the rat. An ultrastructural study. Anat Embxyol 1989; 180: 497-504. (abstract).

27. Hörnblad PY: Embriologycal observations of the Ductus Areteriosus in the guinea-pig, rabbit, rat and mouse. Studies on closure of the Ductus Arteriosus. IV Acta Physiol Scand 1969; 76: 49-57.

28. Olley PM. Coceani F: Mechanism of closure of the ductus arteriosus. En: Godman MJ, Marquis RM, eds. Paediatric Cardiology. Heart disease in 
the newborn. New York: Churchitl Livingstone, 1979: 15-24.

29. Coceani F, Olley $P M$ : The response of the ductus arteriosus to prostaglandins. Canad J Physiol Phatm 1973; 51; 220-225 (citado en Olley 1979).

30. Sharpe G1. Thatme $B$, tarsson $S$ : Studies on closure of the ductus arteriosus. XI Ductal closure in utero by a prostaglandin synthetase inhibitor Prostaglandins 1974; 8: 363-368.

31. Olley PM, Bodach E, Heaton J. Coceani F: Further evidence implicating $E$-Type prostaglandins in the patency of the lamb ductus arteriosus. Fur J Pharm 1975: $34: 247-250$.

32. Olley PM, Coceani $F$, Bodach E: E-Type prostaglandins. A new emergency therapy for certain cyanotic congenital heart malformations. Circulation 1976; $\$ 3: 728-731$.

33. Starliny $M B$, Ellio $R B$ : The effects of prostaglandins, prostaglandins inhibitors, and oxygen on the closure of the ductus arteriosus, pulmonary arteries and umbilical vessets in vitro. Prostaglandins 1974 8: 187-203.

34. Fujito $T$, Ohtani $N$, Aihara $M$, Nishioka $K$. Fujimoto $Y$ : Comparison off the effects of $\mathrm{Fe}^{+2}$ and $\mathrm{Cu}^{+2}$ on prostaglandin synthesis in rabbit kidney medulla slices. J Pharm Phamnacol 1987; 39: $230-233$.

35. Klevay I.M, Viestenz KE: Abnormal electrocardiograms in rats deficient in copper. Am I Physiol $1981 ; 240$ : H185-199.

36. Prohaska JR, Heller LJ: Mechanical properties of the copper-deficient rat heart. $J$ Nutr 1982; 112: 2142-2150.

37. Fay FS: Guinea Pig ductus arteriosus I. Cellukar and metabolic basis for oxygen sensitivity. Am J Physiol 1971; 221: $470-479$ (citado por Olley 1979).

38. ODell BL, Kiburn KH, MCKenzie WN. Thurston $R J$ : The lung of the copper-defficient rat. Atr J Pathol 1978;91: 413-423. 